Aus dem Anatomischen Institut der Universität Basel

Direktor: Prof. Dr. med. et phil. G. Wolf-Heidegger

\title{
Johann Conrad Brunner in Heidelberg als Hochschullehrer und Therapeut
}

Von Edith KüthmanN

Johann Conrad Brunner wurde am 16. Januar 1653 als Sohn des Schultheißen Erhard Brunner in Dießenhofenim Kanton Thurgau geboren. Seine Eltern erkannten früh die außerordentliche Begabung des Knaben und förderten, unterstützt von JohanN Jaков Wepfer, der später Brunners großer Lehrer wurde, seine Neigung zum ärztlichen Beruf.

Schon mit sechzehn Jahren bezog Brunner die Straßburger Universität, um sich dort unter der väterlichen Leitung des Professors Albert Sebicius, der ihn ganz in seinem Hause aufnahm, dem medizinischen Studium zu widmen. Nach vier Jahren intensiver Arbeit legte er bei seinen Lehrern Sebicius, Salzmann und Mappo das Examen ab und verteidigte mit großem Erfolg seine Dissertation über einen Pygopagen (De foetu monstroso et bicipite), den er selbst seziert hatte; ein Thema, von dem Sche Uchzer später in einem warmen Nachruf sagt: «Hacque singulari themate dexteritatem simul in anatomicis et judicii acumen manifestabat neodoctoribus, ut themata seligant haud trita et crambem non apponunt centies vel millies coctam, atque recoctam (Durch dieses einzigartige Thema bewies er den Studenten nicht nur Gewandtheit und Urteilsschärfe in anatomischen Dingen, sondern gleichzeitig, daß man durchaus nicht immer abgedroschene Stoffe wählen und hundert- und tausendmal gekochten Kohl von neuem aufwärmen müsse). $\aleph^{1}$

Nach bestandenem Examen begab sich Brunner nach Paris zu DU VerNEY, dem zu jener Zeit wohl berühmtesten Anatomen Europas. Bei diesem begann er seine Versuche über die Physiologie des Pankreas. «Tag und Nacht», sagt er, "arbeiteten wir in dem Zergliederungssaal, öffneten die Leichname, und meine verschiedenen mühsamen, anatomischen neuen Versuche mit Ausspritzungen der Gefäße, gefielen ihm sehr wohl...» ${ }^{2}$. Du Verney hätte seinen geschickten und fleißigen Mitarbeiter gern bei sich

1 Scheuchzer.

${ }^{2}$ Aepli, S. 441. 
behalten, doch reiste dieser nach einiger Zeit, veranlaßt durch den englischen Forscher Boles, nach England und Holland zu neuen Studien, bis er 1675 auf Wepfers Ruf über Straßburg, wo er sich noch den Doktortitel holte, nach Dießenhofen zurückkehrte. Hier, in seiner Heimatstadt, ließ sich Brunner als praktischer Arzt nieder, arbeitete aber dabei ständig anatomisch-wissenschaftlich weiter und vollendete 1683 seine Arbeit über das Pankreas. Mit Wepfer verband ihn eine menschlich warme Freundschaft, die noch enger wurde, als er 1678 dessen jüngste Tochter heiratete. Johann Jakob Wepfer war zu jener Zeit wohl einer der begehrtesten Ärzte an süddeutschen und österreichischen Fürstenhöfen. Brunner begann sehr bald seinen vielbeschäftigten und mit Arbeit überlasteten Schwiegervater zu vertreten und machte sich dadurch schnell einen Namen. So erhielt er im März 1685 die erste Einladung an den kurpfälzischen Hof, wo er an Stelle des abwesenden Wepfer die Behandlung des todkranken Kurfürsten Karl übernahm. Offensichtlich schenkte die ganze kurfürstliche Familie dem jungen, klugen Schweizer Arzt ihr volles Vertrauen, denn Brunner blieb, auch nach dem Tod des Kurfürsten, bei Hof sehr wohl gelitten und wurde weiterhin zu allen Beratungen hinzugezogen.

Im Mai des gleichen Jahres wurde Brunner als Mitglied in die Kaiserliche Akademie der Naturforscher (Academia Caesareo-Leopoldina) mit dem Agnomen Herophilus aufgenommen, die ihn verpflichtete, von Zeit zu Zeit Beiträge aus seiner Feder zu liefern.

Das Jahr 1686 brachte Brunners Berufung als Professor an die Universität Heidelberg. Die erste Anfrage dieserhalb erging schon im März dieses Jahres an ihn. Brunner sagte erst nach einigem Zögern zu, da er wegen der seit Karls Tode herrschenden religiösen Intoleranz und der schwebenden Kriegsgefahr Bedenken hegte. Am 3. April 1686 finden sich jedoch in den Annalen der Universität folgende Zeilen: «Senat beschließt, in den auf die Ostermesse zu druckenden Lektionskatalog auch die neu angenommenen medic. professoren, iedoch Brunner nur als designatus, auch infime h. Böger als extr. poëseos prof. einzurücken. ${ }^{3}$

Die Universität feierte am 18. Oktober $1686 \mathrm{ihr}$ dreihundertjähriges Bestehen. Diesen Anlaß hatte der neue Kurfürst, Philipp Wilhelm, benützt, um den unbesetzten dritten Lehrstuhl der medizinischen Fakultät wieder einzurichten, um damit der Universität zu erneutem Aufschwung und Ansehen zu verhelfen.

${ }^{3}$ Winkelmann, S. 222. 
So übernahm Johann Conrad Brunner am 2. Oktober 1686 den Lehrstuhl für Anatomie und Physiologie, sowie Botanik im Nebenfach, bei einer Bezahlung von $170 \mathrm{fl}$. Gehalt, einem Fuder Wein und zwölf Malter Korn neben freier Wohnung. In seiner acht Tage später gehaltenen Antrittsrede führte er aus: "Freund seye mir Hippokrates und Aristoteles und Cartesius und jeder andere; aber die Wahrheit seye mir über alles, und diese gestattet niemalen, daß wir auf die Sprüche irgendeines Orakels schwören. Wir dörfen auch niemals einem Lehrer so hartnäckig anhängen, daß wir von ihm glauben, er seye so voll Weisheit, und es könne ihm nichts Menschliches widerfahren. Aber die Wahrheit müssen wir ergreifen, sie komme her, woher sie wolle, von den Alten oder von den Neuen, von Freund oder von Nichtfreund. Die Erfahrung an sich kann nicht trügen; wenn da ein Betrug vorfällt, so ist es unsere Schuld, entweder weil wir nicht aufmerksam genug waren, oder falsch von der Sache geurteilt haben. Die Vernunft, das edelste Geschenk Gottes, laßt uns ganz frey, aber richtig gebrauchen; denn durch sie allein gelangen wir zur Wahrheit. $)^{4}$

Brunners erste Bemühungen galten dem Ausbau des anatomischen Unterrichtes. Um Sektionen und Demonstrationen durchführen zu können, wandte er sich an den Kurfürsten um Sektionsmaterial, das in Heidelberg, wie an anderen Universitäten, äußerst schwer zu beschaffen war. Der Kurfürst war geneigt, den Bitten Brunners bereitwillig nachzugeben und ihn in jeder Hinsicht zu unterstützen. Und Brunner schreibt infolgedessen in der Praefatio zu seiner Arbeit über die Drüsen des Duodenums: «Ich habe mir jeden Stein aus dem Wege geschafft, damit mir nebst häufiger Zergliederung von Tieren auch die Gelegenheit und die Mittel geboten werden, menschliche Leichname inspizieren zu können. Das Glück war meinem Vorhaben günstig. Als nämlich seine Durchlaucht, der Kurfürst, unser allergnädigster Herr, in seiner Gerechtigkeit und Fürsorge gewahr wurde, daß andere Länder uns überflügelt haben und sich glücklich schätzten wegen der Freigebigkeit und außerordentlichen Huld, mit welcher Kaiser, Könige und Fürsten der Kunst und Wissenschaft sich annehmen, da wollte er selbst, der weiseste Fürsorger unserer Universität, auch auf diesem Gebiete des Studiums nicht zurückbleiben. Ja, sogar damit die Leichname seiner verstorbenen Soldaten eher zur Rettung der Überlebenden dienten, als daß sie den Würmern und der Fäulnis anheimfielen, gestattete er, daß diejenigen, welche im Leben dies nicht gekonnt, so doch nach ihrem Tode zum Schutze des Lebens ihrer

${ }^{4}$ Aepli, S. $452-3$. 
Mitbürger beitrügen. $\aleph^{5}$ So standen fortan die in Garnison stehenden und dort verstorbenen Soldaten Brunner zur Sektion zur Verfügung, was nicht nur den Studenten zugut kam, sondern auch Brunners eigene wissenschaftliche Arbeit förderte. Im Jahre 1687 veröffentlichte er jene Schrift, die seinen Namen für immer in der Medizin verankert hat: Novarum glandularum intestinalium descriptio, in der die später nach ihm benannten Drüsen des Zwölffingerdarms zum erstenmal ausführlich beschrieben worden sind.

Brunners zweite Sorge galt der chemischen Ausbildung seiner Medizinstudenten. So schlägt er, wie die Heidelberger Annalen vom 29. Oktober des Jahres 1687 berichten, vor, daß «den studiosis medicinae - in specie aber, so chimiam liben - ein Laboratorium eingeräumt werde». ${ }^{6}$ Und weiter heißt es : «Der med. fac. wird dazu nov. 8. ein hinterhaus eingeräumt. » Die gleichen Annalen verzeichnen im Juli 1687: «Georg Frank, med. et phil. dr. lädt als Dekan der med. fac. zu botanischen exkursionen in die umgebung Heidelbergs ein, welche dr. med. J.K. Brunner und der aufseher des hortus medicus, chirurg P. Carre, leiten werden. ${ }^{7}$ Und Frank lobt in einem Einladungsschreiben zu dieser Exkursion Brunners ausgezeichnete Pflanzenkenntnis. Brunner selbst hat der Botanik wohl nur geringes Interesse entgegengebracht; jedenfalls existieren keinerlei Arbeiten, die darauf hindeuten, daß er sich außerhalb der Pflicht mit pflanzlichen Studien beschäftigt hat.

Brunners Verhältnis zu seinen Kollegen sowie zu den Studenten muß außerordentlich herzlich gewesen sein. Aepli beschreibt sein ausgeglichenes Wesen, das ihm überall Freunde schuf, folgendermaßen: «Brunner bedörfte der elenden niederträchtigen, höchst ärgerlichen und Wissenschaft und Sitten verderblichen Professorenstreiche nicht, um sich von Studenten gelobt zu sehen, und seiner Schule Rekruten zu verschaffen. Er war ein edelmüthiger, friedliebender Mann, der mit Gelehrsamkeit nicht prahlte; nicht den Charlatan machte, und jeder Niederträchtigkeit unfähig war.» ${ }^{8}$ 1687 wurde Brunner die freie Arztstelle in der Garnison zu Philippsburg angetragen, die er trotz den Bedenken, die Rektor und Senat der Universität aus Furcht, die Professur möchte darunter leiden, dagegen hegten, gern annahm, indem er die Nachbehandlung einem von ihm eingelernten

5 Nebel, Oratio de vita et meritis professorum medicinae.

6 Winkelmann, S. 225.

${ }^{7}$ Winkelmann, S. 225.

${ }^{8}$ Aepli, S. 454. 


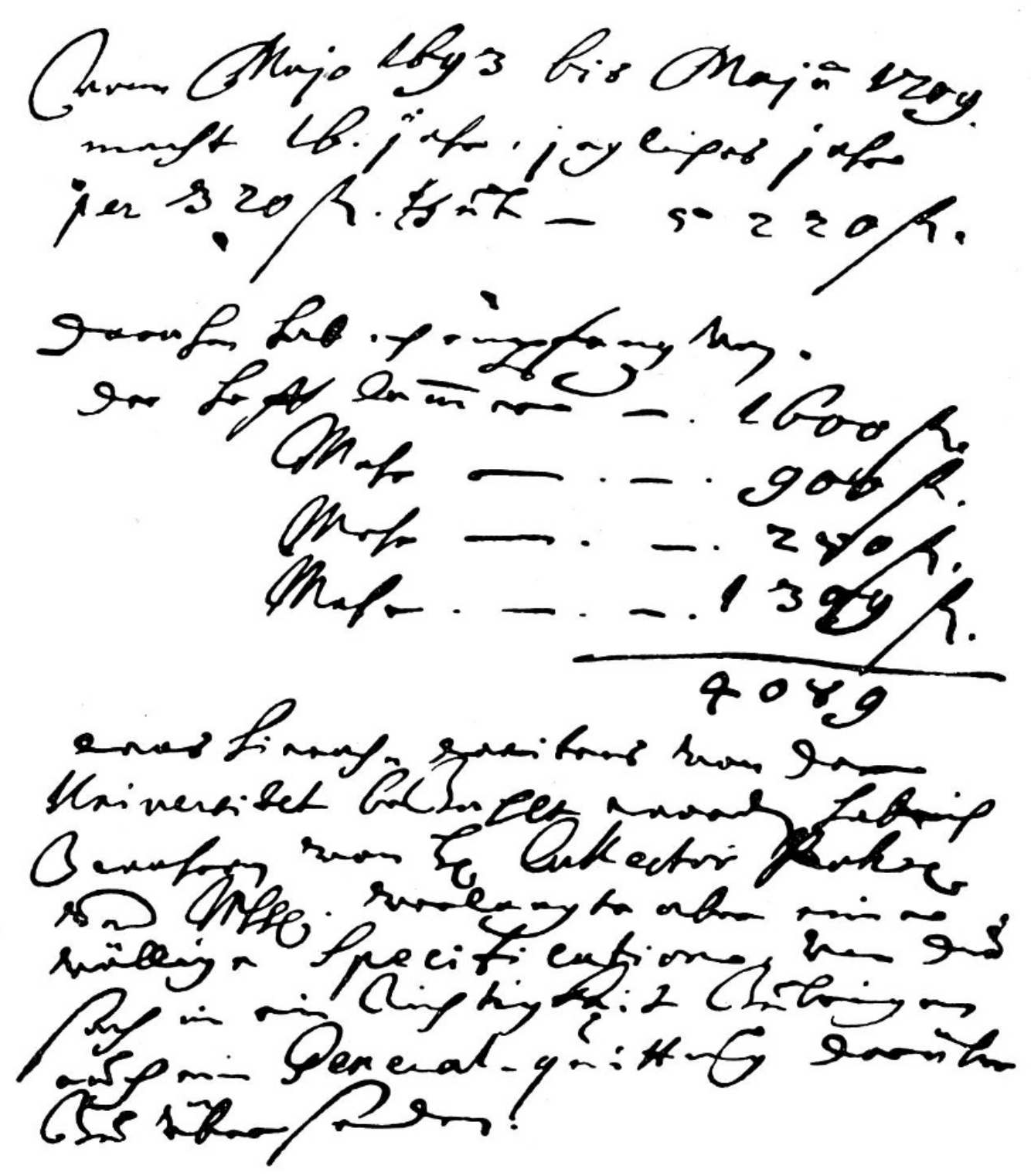

Abb. 1. Spezifizierte Rechnung Johann Conrad Brunners über Gehaltsausfall vom Mai 1693 bis Mai 1709

Arzt überließ, der ihn in leichten Fällen in seiner Abwesenheit auch ohne weiteres vertreten konnte.

Im Jahre 1688 brach der Orléanssche Krieg über die Pfalz herein, der das Land in furchtbarer Weise verwüstete. Am 24. Oktober 1688 zogen französische Truppen auch in Heidelberg ein. Der Rektor der Universität, Fabricius, unterzeichnete die Kapitulationsurkunde mit und erreichte 
durch folgenden Artikel gesetzliche Schonung der Universität: «... que l'université avec toutes les personnes en dépendantes de même que la ville et les bourgeois et habitans d'icy seront maintenus dans tous droits privilège libertéz et immunitéz et que particulièrement la dite université demeurera dans la paisible possession de ses biens et revenus tant dedans que dehors le pays, sans y estre troubléz ou empechéz...» ${ }^{9}$.Dennoch setzten Zerstörung und Vertragsbruch allen friedlichen wissenschaftlichen und künstlerischen Bestrebungen ein rasches Ende. Der Kurfürst hatte die Stadt verlassen; die Universität war so gut wie geschlossen, da die meisten Professoren geflohen oder dem Ruf an andere Universitäten oder Fürstenhöfe gefolgt waren. Brunner hatte den Sturm vorausgesehen und seine Familie rechtzeitig nach Dießenhofen geschickt. Er selbst blieb der Form nach Professor in Heidelberg und Garnisonsarzt in Philippsburg; den Rang eines Oberarmeearztes für das ganze kaiserliche Heer, den man ihm hatte verleihen wollen, lehnte er ab. Er befand sich aber von nun an fast ständig als Consiliarius auf Reisen, wie aus folgendem Bericht hervorgeht: «Ex quo dulcissima quondam Musarum castra Palatina in horrida Martis mutata reliqui, incertus loci praxeos et hinc variorum itinerum cursu per Helvetiam, Alsatiam, Imperium Germanicum mox ad Aulam Caesaream, usque inde per Hassiam, Duesseldorpium terna vice, Berolinum etc. delatus varias hinc inde Aulas aegrotantium Principum, Electorum et Regum causa visitavi... (So verließ ich die Pfalz, die einst so wunderbaren Stätten der Musen, die jetzt in die verheerenden Stätten des Mars verwandelt worden sind, und reiste als Pilger zu wechselvoller ärztlicher Tätigkeit durch die Schweiz, das Elsaß, an den kaiserlichen Hof, und von da nach Hessen. Nach dreimaligem Aufenthalt in Düsseldorf wurde ich nach Berlin verschlagen; dann besuchte ich wieder verschiedene andere Höfe kranker Fürsten und Könige.... ${ }^{10}$.

Der zunächst noch in Heidelberg verbliebene Professor Frank, Brunners Kollege, schrieb 1689 an diesen: «Wi isch ? Wi gots Herri ? By Gott Bluot schlächt! So müssen wir arme heidelbergische Universitäts Fixsternen nunmehr zu Planeten werden, und bei diesem elenden Winter in dem römischen Reich herumrollen.» ${ }^{11}$

Die Lage der Universität verschlechterte sich in kurzer Zeit so sehr durch die Belastungen, die durch die feindlichen Truppen entstanden, da $\beta$ die

${ }^{9}$ Winkelmann, S. 226.

${ }^{10}$ Schwab, S. 109.

11 Aepli, S. 458. 
Professoren vom Rektor ersucht werden mußten, nicht nur zunächst auf Gehalt zu verzichten, sondern auch einen Teil dazu beizutragen, daß die Universität nicht völlig dem Verfall anheimgegeben werde. Der Theologe FABRICIUS, der als letzter Getreuer in Heidelberg aushielt, schrieb im Oktober 1689 an Brunner, in der Hoffnung, dieser würde zurückkommen und für das Jahr 1690 die Rektorwürde übernehmen : «Ist noch einige Hoffnung übrig, daß sie die vorigen Wohnungen zu Heidelberg, welche nunmehr mit verwundeten Bayern belegt sind, besetzen wollen? Der berühmte Frank hat uns auch verlassen und ist von dem Churfürsten in Sachsen und der Universität Wittenberg angezogen worden. Von ihnen sagt man, daß sie sich dem Landgrafen von Cassel und der Schule Marburg ergeben hätten. Wir verlieren also alle Lehrer. Mich haben die Holländer wollen. Aber ich verlasse den Posten nicht, den mir die Vorsehung angewiesen hat, solange ich etwas nützen kann. Doch kann ich hier den Winter nicht zubringen, sondern gehe nach Frankfurt, ausgenommen Sie als für das künftige Jahr zu erwählender Rektor magnificus, kommen hieher. Schreiben Sie uns, ob wir diesen Trost haben können. ${ }^{12}$

Brunner lehnte ab und antwortete in folgendem Brief: «Was meine Wenigkeit betrifft, habe ich mich in keine weitere Obligation eingelassen und halte mich, soviel vermeyne, extra teli jactum, solange bis die obschwebenden Kriegsgefahren ihr Ende erreicht haben. Gleichwie nun die Zeit einen jeden lehren wird, was für Maßregeln zu nehmen, also hoffe, eine hochlöbliche Universität werde mir nicht übelnehmen, wenn ich bis dahin meine endliche Resolution mir vorbehalten und mich nach der Zeit richte...» ${ }^{13}$.

Philipp Wilhelm starb 1690. Brunner erfreute sich jedoch so großer Gunst bei Hof, daß er bei nächster Gelegenheit vom neuen Kurfürsten, der seine Residenz in Düsseldorf aufgeschlagen hatte, gerufen und in seinem Amt als Leibmedicus bestätigt wurde. So weilte Brunner, wenn er nicht auf Reisen war, bei Johann Wilhelm, der sich alle erdenkliche Mühe gab, den berühmten Arzt in seiner Nähe zu halten. 1695 ernannte er ihn zum Professor primarius der medizinischen Fakultät Heidelberg, was eine Aufbesserung von Brunners Gehalt auf $280 \mathrm{fl}$. mit sich brachte; da es jedoch der Universität durch Jahre hindurch finanziell an allen Mitteln gebrach, mußte Brunner in unendlich vielen Reklamationsschreiben, die im Archiv der Universität Heidelberg heute noch einzusehen sind (siehe Abb. 1 bis

12 Aepli, S. 458-9.

${ }^{13}$ Aepli, S. 465. 


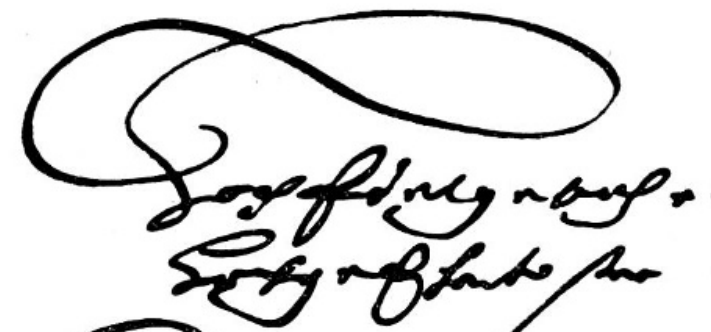

27

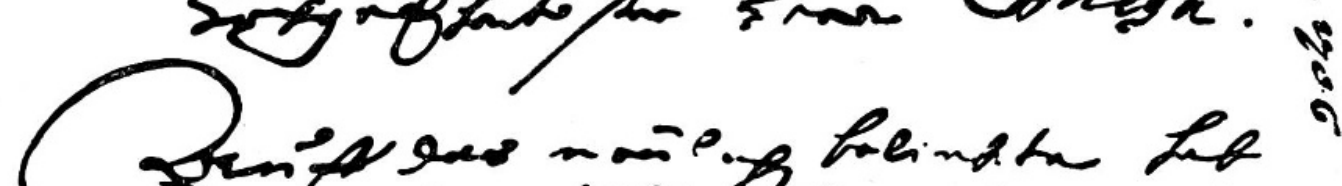

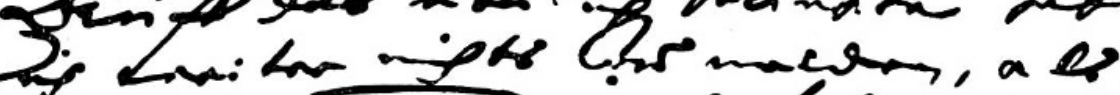

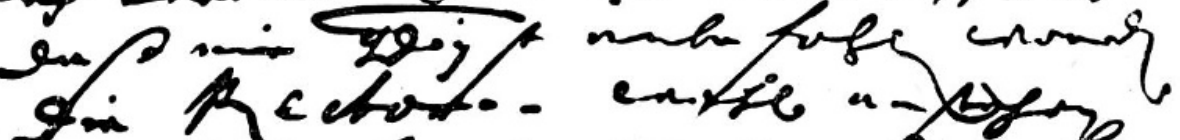

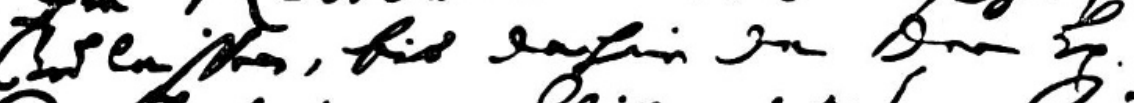

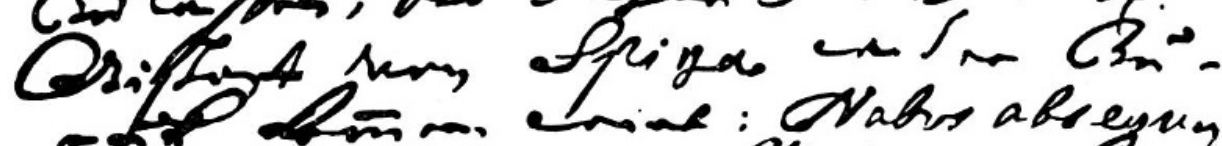

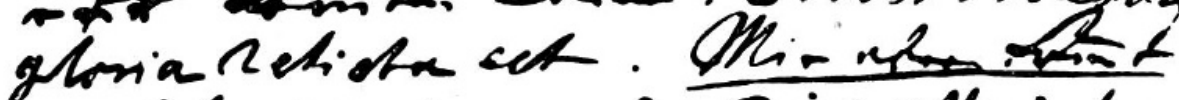

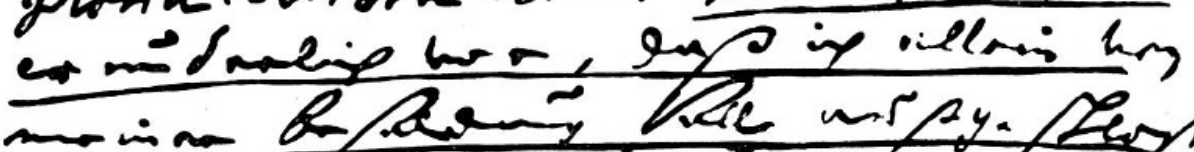

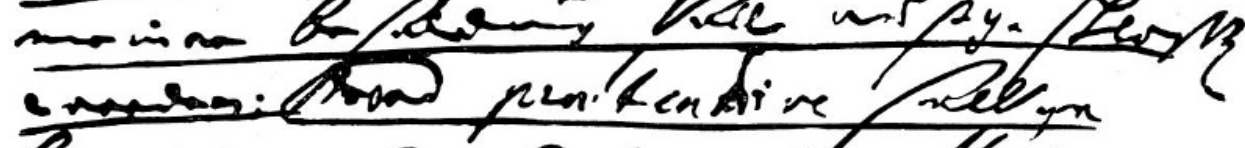

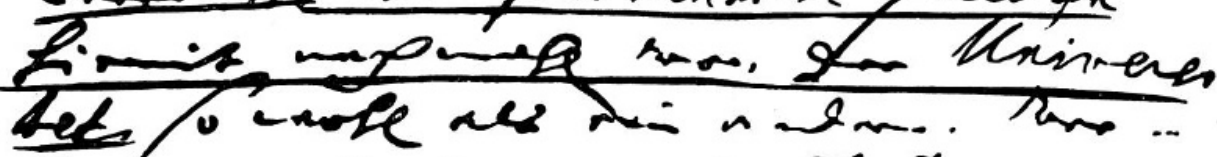

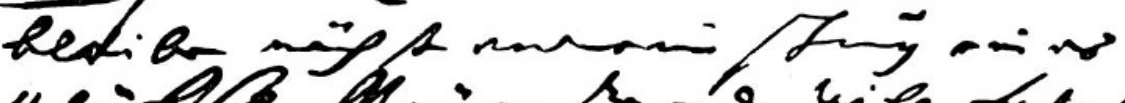

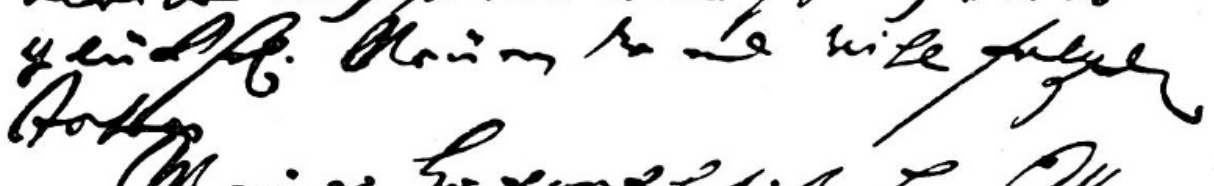

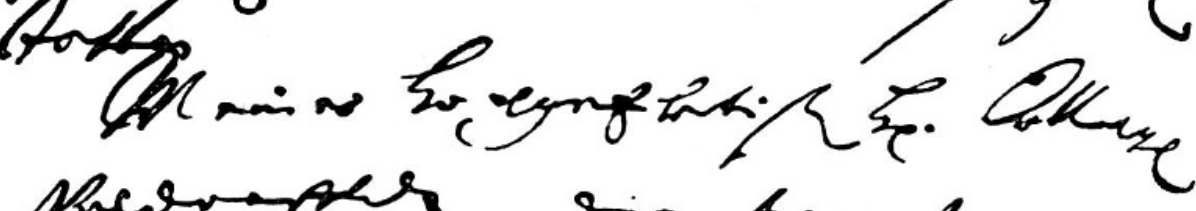

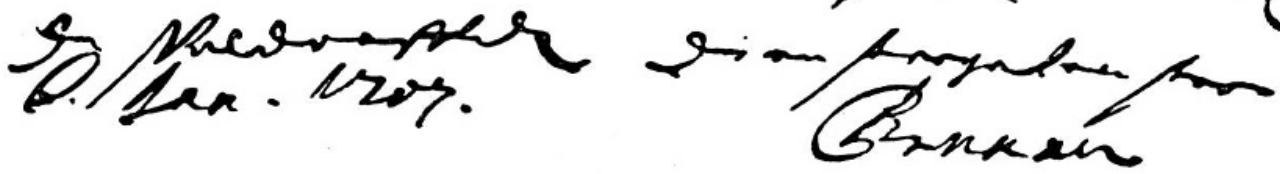

Abb. 2. Brief Brunners aus Düsseldorf vom 6. Januar 1707 mit der Forderung, sein Gehalt nachzuzahlen 
4), seine Ansprüche wieder und wieder geltend machen, um zu seinem Recht zu kommen. So schreibt er in einem, vom 6. Januar 1707 aus Düsseldorf datierten Brief unter anderem die bitteren Sätze: «... Nobis obsequi gloria relicta est. Mir aber kommt es wunderlich vor, daß ich allein von meiner Besoldung solle ausgeschlossen werden und praetendire selbige hier mit nochmals von der Universitet, sowol als wie andere... ${ }^{14}$ (siehe auch Abb.2). Den Kampf setzte später nach seinem Tode seine Witwe fort, ob mit Erfolg, ist nicht zu klären.

Dennoch war Brunners Zuneigung und Treue zum kurpfälzischen Hause so groß, daß er einen Ruf an die Universität Leyden, der ihm durch SpaNHEMIUs im Jahre 1697 zuging, ablehnte, und zwei Jahre später, den Holländern, die immer noch hofften, den berühmten Mann für ihre Hochschule gewinnen zu können, ein zweites Mal eine abschlägige Antwort zusandte und seine Beweggründe zu dieser Absage nannte. V. SснметтA antwortete Brunner darauf in folgendem Schreiben: «Dessen beliebtes vom 29. Augstm. habe wol erhalten, und daraus die Ursachen ersehen, warum Mnhhr. Rath die zu Leiden offerirte Professionem medicam primariam jetziger Zeit excursirt. Ich begreife dieselben und kann nichts sagen, sowol gegen das Attachement an einen so gnädigen Herrn, als auch an die Churpfalz, und ex cineribus wieder hervorkommende Heidelbergische Universität, die solcher Patronen und Vorsprecher der reformirten Religion, zugleich mit dem gesamten Kirchenwesen in der Pfalz sehr nötig hat. Gott wolle Mnhhrn. in so löblichen Vorhaben segnen. $)^{15}$

Brunner blieb weiterhin meistenteils auf Reisen, und wir finden ihn in diesen Jahren bei den verschiedensten weltlichen und geistlichen Herren, denen er ärztlichen Beistand leistete.

Die Universität hatte sich nach ihrer zweiten und völligen Zerstörung im Jahre 1693, der auch die medizinischen Räume wie das nosocomium und außer diesen die ganze Bibliothek zum Opfer gefallen waren, dem Rufe des Theologen Fabricius folgend, nach und nach, und soweit sie in ihren Mitgliedern noch bestand, wieder in Frankfurt zusammengefunden und übersiedelte 1698, als Johann Wilhelm endlich aus seinen Herzogtümern Jülich und Berg in sein gänzlich darniederliegendes und erschöpftes Land zurückkehrte, mit ihm in die neugewählte Residenz Weinheim. Brunner wurde für dieses Jahr zum Rektor magnificus bestimmt, und in den Uni-

${ }^{14}$ Originalbrief, Archiv der Universität Heidelberg.

${ }^{15}$ Aepli, S. 477. 
versitätsakten findet sich folgender Passus: «15. april. Joh. Conr. Brunner kurf. Rat und leibmedicus, h. t. univ. Palat. rektor, schreibt aus Düsseldorf seinen Kollegen, daß der kurf. um die univ. zu repariren von ihnen vorschläge erwarte. Auf befehl des kurf. habe er auch an den Dän. leibmedicus dr. Frank geschrieben, der seine wiederkunft zwar nicht abschlage, aber zunächst über die einkommensverhältnisse unterrichtet sein wolle.» ${ }^{16}$

Da Brunner trotz seines Amtes fast die ganze Zeit auf Konsultationsreisen war, ließ er sich von dem Rechtswissenschaftler FLECK von RosenECK in seiner Eigenschaft als Rektor vertreten. 1700 kehrte die Hochschule mit ihrem Lehrkörper, der nur noch aus vier Dozenten bestand, endlich nach Heidelberg zurück; jedoch konnte sie sich lange nicht von den schweren Schäden, die sie erlitten hatte, erholen. Brunner, der 1702 wiederum Rektor war und an seiner Statt Morass bat, die Geschäfte zu führen, schreibt in einem Brief aus Düsseldorf: «Ich hör und sehe nichts mehr von unserer Universitett.» Und er bittet den Syndikus der Universität, Clokter, an den der Brief gerichtet ist, ihm doch mitzuteilen ... « wie es mit den Universitettssachen stehe, ob die Hrn. Professores zusammenkommen und ihre Besoldung wieder woher ziehen? Ich hab immer gehofft, diesen Sommer hinaufzukommen, weiß aber nicht, ob etwas daraus werden wird.» ${ }^{17}$

Trotz seiner häufigen Abwesenheit stand Brunner der Universität mit Rat und Tat zur Seite. Dem Wunsche seines Landesherrn entsprechend, bemühte er sich sehr, berühmte Professoren aller Fakultäten nach Heidelberg zu ziehen. So suchte er den Lehrstuhl für reformierte Theologie durch einen Züricher Theologen zu besetzen. Offensichtlich hat Brunner später mit der überwiegend katholischen Einstellung seiner Kollegen zu kämpfen gehabt, da mit Karls Tod die protestantische Linie der Pfalz erloschen war, und mit der katholischen Linie Pfalz-Neuburg die Jesuiten stark in das geistige Leben eingriffen.

Da Brunner als Rektor, als «vir nobilis», nicht nur in "arte medica», sondern auch «in rebus philosophicis» mitzusprechen hatte, benützte er die Gelegenheit, statt der bisher gelehrten Philosophie des Aristoteles der Lehre Descartes' zum Durchbruch zu verhelfen. 1704 bekleidete Brunner erneut das Amt des Rektors und vermittelte mit glücklicher. Hand den Ankauf einer neuen Bibliothek, die der Kurfürst auf sein Anraten aus dem Besitz des verstorbenen Leydener Professors Graevius erwarb und der

16 Winkelmann, S. 231.

${ }^{17}$ Originalbrief, Archiv der Universität Heidelberg. 

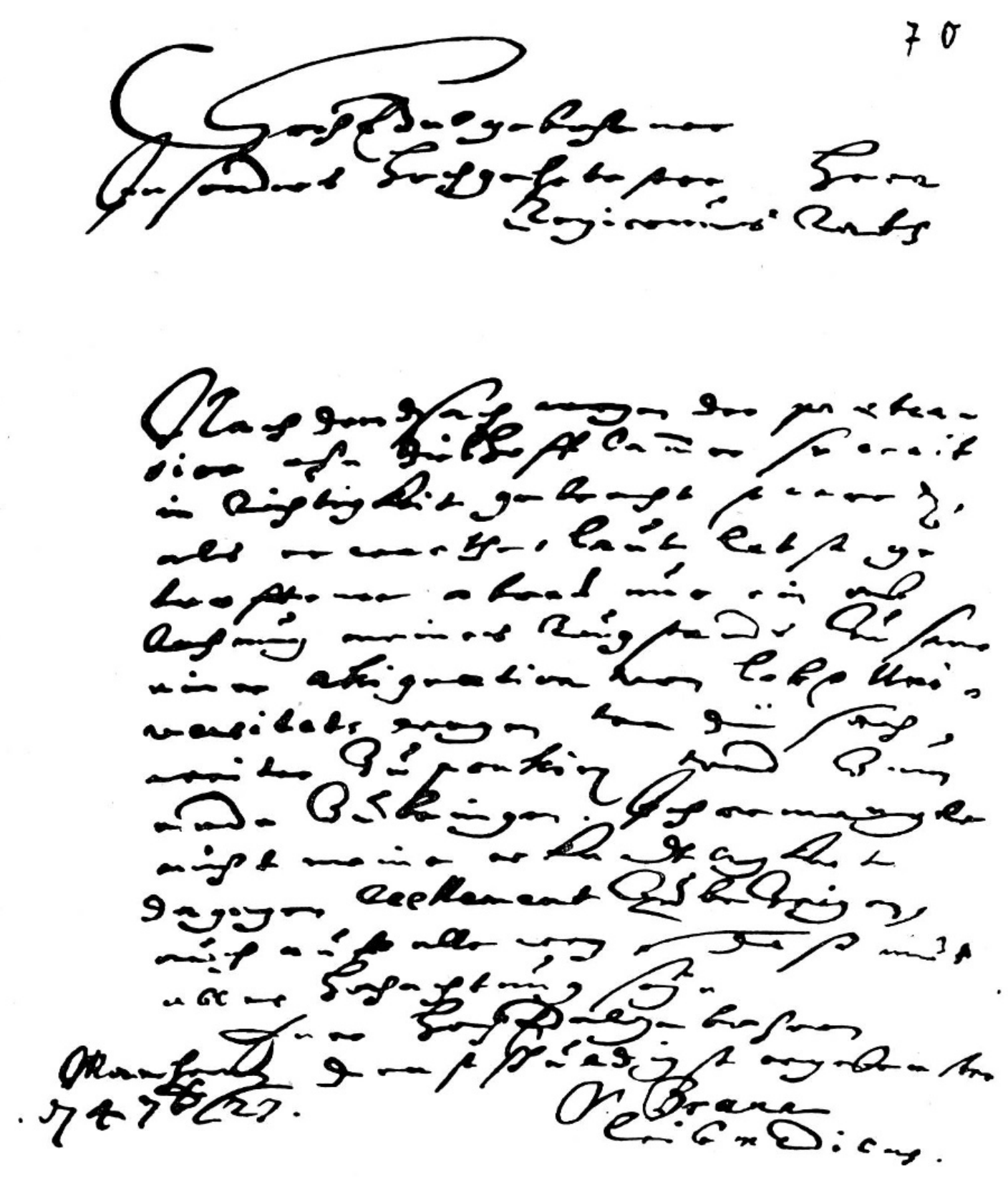

Abb. 3. Brief an den Regierungsrat wegen Rückstand der Besoldung aus dem Jahre 1727

Universität zum Geschenk machte. In den nächsten Jahren bat Brunner, der noch immer einziger Vertreter der medizinischen Fakultät war, den Senat, Daniel Nebel aus Marburg als Extraordinarius zu seiner Entlastung zu berufen, da nun acht Jahre nach der Rückkehr der Universität «collegia, actus, examinationes et promotiones» noch immer nicht vorgenommen 
werden könnten ${ }^{18}$. 1711, als Brunner das Leben des Kurfürsten, der einen apoplektischen Anfall erlitten hatte, um fünf Jahre verlängern konnte, wurde er zum Dank für seine ärztliche Hilfe und seine großen Verdienste in den Adelsstand erhoben und mit dem Besitz Hammerstein im Bergischen belehnt. Brunner führte von nun an den Namen Freiherr von Brunn von Hammerstein. 1716 erlag Johann Wilhelm einem zweiten apoplektischen Anfall. Brunner blieb jedoch als Leibmedicus in kurpfälzischen Diensten und wurde von Johann Wilhelms Nachfolger, Karl Philipp, bestätigt. So hielt sich Brunner zunächst in Innsbruck auf, wo Karl Philipp in der ersten Zeit residierte. 1718 begleitete er seinen Landesherrn bei dessen Einzug nach Heidelberg. Enttäuscht stellte er dort fest, daß während seiner Abwesenheit an der Universität große Veränderungen vor sich gegangen waren und die von ihm eingeführte Cartesianische Lehre unter dem Einfluß der Jesuiten aus dem Lehrplan wieder gestrichen und durch die naturfremde Scholastik ersetzt worden war.

Brunner hat sich in den letzten Jahren seines Lebens nicht mehr viel um die Hochschule kümmern können, da er als Konsiliarius zu stark in Anspruch genommen war.

1720 folgte er Karl Philipp nach Mannheim, wohin dieser mit dem ganzen Hof übersiedelte; dort beschloß er am 2. Oktober 1727 sein arbeitsreiches und erfolggekröntes Leben.

Die Annalen der Universität Heidelberg berichten aus diesem Jahr, daß mit Brunners Tod auch die Professur erloschen sei, weil «keine dem studio medico obligende scholaren sich allhier befinden. ${ }^{19}$

Johann Conrad Brunner ist zweifellos eine der bedeutendsten Persönlichkeiten gewesen, die der medizinischen Fakultät der Universität Heidelberg angehört haben. BEcKER sagt in seiner Rektoratsrede vom Jahre 1876 in diesem Zusammenhang: «In der Geschichte der Medizin aber, wir müssen es offen bekennen, wird die Heidelberger medizinische Fakultät vor diesem Jahrhundert kaum genannt. Nimmt man Erasts Schriften gegen Paracelsus und einige Arbeiten von Brunner aus, so dürfte alles andere schon jetzt der Vergessenheit anheimgefallen sein oder doch nur eine lokale Bedeutung haben ${ }^{20}$, und damit legt er Brunners hervorragende Stellung fest, die ihn als einzelne Persönlichkeit aus einem Zeitraum von mindestens dreihundert Jahren allein herausgehoben hat.

18 Winkelmann, S. 243.

19 Cod. Heid. 386, 46.

20 Becker, Zur Geschichte der medizinischen Fakultät in Heidelberg, 1876, S. 6-7. 
Auf Brunners Bedeutung als Forscher auf anatomischem und physiologischem Gebiet ist in verschiedenen Arbeiten hingewiesen worden ${ }^{21}$. Ich lasse sie deshalb im Rahmen dieser Besprechung unberücksichtigt. Die Wissenschaft sieht mit gewissem Bedauern darauf, daß Brunners Kräfte durch seine ärztlich-praktische Tätigkeit zum großen Teil gebunden und damit der reinen Forschung entzogen waren. "Pauca dedit argumenta anatomici, sed eximia ${ }^{22}$, sagt schon HALLER in diesem Zusammenhang.

Der heutigen Klinik jedoch hat Brunners «Leibmedizinerei», deren zum Teil überraschend modern anmutende Erkenntnisse in den Observationes der Ephemeriden der Academia Caesareo-Leopoldina veröffentlicht sind, Unschätzbares geschenkt. Es handelt sich hier um klinische Berichte, häufig mit ausführlichen pathologisch-anatomischen Befunden, die in ihrer Auswertung weit über die damalige Zeit hinaus und bis in unser Jahrhundert hineingreifen.

Um Brunners therapeutische Leistung wirklich und in ihrem vollen Umfang zu verstehen, sei hier der Stand der Medizin des 17. Jahrhunderts kurz gestreift und beleuchtet: Grundsätzlich lassen sich wohl drei Hauptrichtungen der praktischen Heilkunde jener Zeit unterscheiden: die Iatrochemie als die stärkste, unter ihrem Oberhaupt Sylvius (Franz DE LA BoE), Professor in Leyden, die alle Lebensvorgänge auf chemische Grundlagen zurückzuführen suchte und dabei Speichel, Pankreassaft und Galle eine hervorragende Bedeutung im Stoffwechselgeschehen zumaß.

Daneben entwickelte sich die Schule der Iatrophysiker, die, auf HARveYs fundamentaler Entdeckung des Blutkreislaufes fußend, ein exaktes mechanisches System ausarbeiteten, das zwar naturnahe, aber letztlich unfruchtbar und tot war, indem es den menschlichen Organismus der Maschine gleichzusetzen suchte.

Als dritte Richtung der Medizin läßt sich die empirische nennen. Ihr gehörten die großen Praktiker des 17. Jahrhunderts an, die, fern von Hypothese und Spekulation, nur den Grundgesetzen des Hippokrates folgend, ihre Erkenntnisse durch geschärfte Aufmerksamkeit, Beobachtung und Erfahrung der Natur abgewannen.

Einer im eigentlichen Sinne freien, ungehemmten Entwicklung der Naturwissenschaften stand ihre enge Bindung an philosophische Systeme entgegen. Die aristotelische Syllogistik, die lange Zeit die Grundlage allen

21 Siehe die Arbeit von Ole Christian Zimmermann, 1945.

22 Bibl. anat., Band I, S. 595. 
medizinischen Denkens gewesen war, wurde zwar im 17. Jahrhundert weitgehend durch die exakt-mathematische Lehre Descartes' verdrängt, jedoch bedeutete auch diese für die Medizin weder wirklichen Umschwung noch Gewinn, da sie, von falschen Voraussetzungen ausgehend, falsche Relationen knüpfte und falsche Schlüsse zur Folge hatte. Die teilweise gleichzeitig gelehrte Scholastik schloß jede logische naturwissenschaftliche Auseinandersetzung überhaupt aus oder erstickte alle Ansätze dazu schon im Keime.

Den Voraussetzungen entsprechend, die die entweder vorwiegend chemiatrische oder iatrophysikalische Anschauung der Medizin schuf, stand es um die Therapie schlecht. Wepfers Generation hatte eben begonnen, die anatomischen und pathologisch-anatomischen Grundlagen zu erarbeiten, auf denen die «Klinik» weiterbauen konnte; Chemie, Botanik, Toxikologie und Pharmakologie standen am Beginn ihrer Entwicklung. Die Chirurgie lag zum größten Teil noch in den Händen der Bader und wurde von der eigentlichen Ärzteschaft weitgehend vernachlässigt.

So blieb die Behandlung, jedenfalls die erfolgreiche Behandlung, fast ausschließlich an die Persönlichkeit des Arztes gebunden, der durch die Fähigkeit, physische Beschwerden großenteils auf psychischem Weg zu beeinflussen und zu bessern, die große Lücke der fehlenden Hilfsmittel zu ersetzen hatte. Und dieser Tatsache ist es wohl zu danken, daß hier ärztliches Können immer mit wirklich echter und warmer Menschlichkeit zusammenfiel.

Brunner war, soweit es sich um theoretische Dinge handelte, strenger Anhänger Descartes', dessen Lehre von der Seele des Menschen, die in der Zirbeldrüse verankert sei und von dort durch die Lebensgeister Botschaften an den Leib aussendet und empfängt, er voll anerkannte und die sich in seinen anatomischen Arbeiten an vielen Stellen widerspiegelt. Als praktischer Arzt und Therapeut jedoch vertraute Brunner allein seinem Auge und der Erfahrung. Brunner und von Muralt sagen von ihm: «Die glücklichen Kuren, die ihn zum ,berühmtesten medicum der Welt' (nach Aepli) machten, mußte er mit der kümmerlich quacksalberischen, auf unzulänglichen oder falschen pathologischen Voraussetzungen basierenden Therapie seiner Zeit erzielen; er hat sie offenbar mit Skepsis beherrscht, mit Klugheit angewendet und daneben hauptsächlich suggestiv, diätetischhygienisch gewirkt. ${ }^{23}$ Brunners überlegte und vom modernen Gesichts-

23 Brunner und von Muralt, S. 152. 
punkt ausgezeichnet begründete Therapie mit einfachen, aber wirksamen Mitteln zeichnet sich außerordentlich eindringlich an seiner eigenen Krankengeschichte ab, die er im Alter von siebzig Jahren als Observatio niedergeschrieben und veröffentlicht hat ${ }^{24}$. Ich lasse sie hier im Zusammenhang folgen:

Lac natura omnibus destinatum alimentum, salutare promittit, quoque medicamentum: partibus sanis alendis cum sit abtum aegrotantibus quoque reficiendis erit idoneum. Succum praebet mitem, natura gratum balsamicum, nulli non acceptum, nisi organum gustus vitiarit lautitiis, saporibus, qui seculi mos est multifariis et irritamentis pravam consuetudinem contraxerit.

Lactis beneficium cum in aliis, cum viris Celeberrimis, tum in me ipso expertus sum proprio experimento, quod cum aliis communicare publici interesse putavi.

Medicus septuaginta annorum, natus parente podagrico, matre nephritica, non multos, si vis, nullos commisi excessus, studiis addictus, a juventute integra usus sum valetudine; itinera per Germaniam, Galliam, Angliam atque Bataviam studia secutus, bene tuli. Primordia nephritidis sensi anno aetatis vigesimo quarto $\mathbf{P a}$ risiis, ubi multum fabuli flavi minci ac tenuis, postea crassioris, cum alias tum praesertim quanto bibi meracius. Provectior aetate lapillos genui in renibus et excrevi majusculos, cum solitis doloribus, praesertim a potu vini albi, Rhenani, Mosellani, Nicrini, multiplici experientia edoctus. Annum quinquagesimum supergressus podagrae quoque insultus sensi primum ex pedis dextri radice pollicis; abhinc sinistri; postea genua incessit; inde manus, cubitos humerosque; collum deni-
Die Milch, die ein von Natur aus für uns alle bestimmtes Nahrungsmittel ist, ist auch für Heilzwecke zu verwenden; da sie zur Ernährung der Gesunden dient, wird sie auch für Kranke und Genesende geeignet sein. Sie ist so milde und balsamisch im Geschmack, daß sie von allen Menschen, die ihren Gaumen nicht durch die zu unserer Zeit üblichen Leckereien und Reizmittel verdorben haben, gern genommen wird.

Die Wohltat der Milch habe ich an allen möglichen berühmten und weniger berühmten Leuten ausprobiert, und schließlich auch an mir selbst. Das Ergebnis wird, denke ich, auch die Allgemeinheit interessieren.

Ich bin ein siebzigjähriger Arzt, Sohn eines gichtbelasteten Vaters und einer Mutter, die unter Nierenschmerzen litt. Ich habe wenige, eigentlich gar keine Ausschweifungen begangen, habe fleißig gearbeitet und bin von Jugend an gesund gewesen. Ohne Beschwerden führte ich meine Studienreisen nach Deutschland, Frankreich, England und Holland durch. In meinem vierundzwanzigsten Lebensjahr verspürte ich zum ersten Mal in Paris Nierenschmerzen, wobei ich viel gelben, zunächst feineren, später gröberen Grieß im Urin ausschied, besonders nach Weingenuß. In fortgeschrittenem Alter bildeten sich Steinchen in den Nieren, die unter den üblichen Schmerzen abgingen, und zwar, wie ich oft erfahren habe, meistens dann, wenn ich weißen Rhein-, Mosel- oder Pfälzer Wein trank. Nach meinem fünfzigsten Jahr machten sich die ersten Gichtanfälle bemerkbar. Zuerst an der Wurzel der rechten großen Zehe; dann

${ }^{24}$ Observatio Experimentoum circa Podagram cum Nephritide, Vol. I. Autor. Ann. 1727, Übersetzung in Anlehnung an AEPLI. 
que et nullam non partem varie afflixit, per intervalla primo longiora, postea breviora, et me muneri obeundo tantum non inhabilem reddidit; quin interiora identidem affici, stomachum praesertim, timide sensi. Paroxysmum podagricum plerumque excepit nephriticus, quo calculum exclusi, et fere numquam non fabulam cum urina reddidi, futuri paroxysmi nuntia. Varia tentavi nec sine successu. Tandem vero metu graviorum cogitatio subiit de lacte, communi podagricorum, quos intemperies gulae non subjugavit, solatio. Post multas difficultates nostro seculo insuperabiles vicit tandem amor sanitatis tam propriae, quam proximi; indignum ratus medicus nemini prodesse et pane vesci seu inutile terrae pondus.

Auspicatus sum curam lactis mense aprili quem agimus MDCCXXIII cum bono Deo et continuavi hucusque XXV. octobr. quo haec scribo eo cum successu ut ab eo tempore neque podagrae, neque nephritis dolorem senserim, qui ante immunis numquam expeditus fui. Appeto; digero citra molestiam; non sitio; egero debito: et quod mirum est ne micam quidem sabuli ab omni tempore video; nec haemorrhoides, quas ante hac habui; dormio bene; pulsum quem olim habui celerem, nunc tardum, plenum et fortem percipio; habitus corporis augescit, et vires crescunt; verbo: integra per Dei gratiam nunc fruor valetudine; melius quoque ambulo, pedibus manibusque utor, nisi quod partes affectae ad eam integritatem hactenus quidem non redierim atque robur, quod ante magnum habui, ad actiones peragendas. Quae quidem bona, Deo benedicente lacti debeo. Cuius vaccini recens mulcti mane //. an der linken; darauf am Knie, in den Händen, Ellenbogen und Schultern; endlich auch im Hals; kein Körperteil blieb verschont. Die Abstände zwischen den einzelnen Anfällen waren zuerst groß, später immer kleiner, jedoch konnte ich immer noch arbeiten. Schließlich wurden auch die inneren Organe befallen, besonders der Magen, dabei gingen im allgemeinen Nierenschmerzen vorauf mit Grieß- und Steinabgang; dies war das Zeichen für den bevorstehenden Gichtanfall. Ich habe Verschiedenes mit Erfolg versucht. Jedoch kam mir schließlich aus Furcht vor größerem Übel die Milch in den Sinn, das allgemeine Hilfsmittel aller noch nicht zu verschleckten Gichtkranken. Nach vielen Schwierigkeiten, die in unserem Jahrhundert unüberwindlich zu sein scheinen, siegte schließlich die Liebe zu meiner eigenen Gesundheit und der meines Nächsten, denn ich hielt es für unwürdig, niemand zu nützen und doch Brot zu essen bzw. ein unnützer Gast auf dieser Erde zu sein.

Ich begann mit der Milchkur im April 1723 und setzte sie bis zum 25. Oktober, dem Tag, an dem ich dieses schreibe, ununterbrochen und mit solchem Erfolg fort, daß ich seither weder Gicht- noch Nierenschmerzen habe, von denen ich doch vorher niemals ganz frei gewesen war. Ich habe Lust zum Essen und beschwerdelose Verdauung; ich habe keinen Durst, der Leib öffnet sich regelrecht; und was mich am meisten wundert, ich sehe seit dieser Zeit keine Spur von Sand; außerdem sind meine Hämorrhoiden ganz zurückgegangen. Ich schlafe gut; mein schneller Puls ist langsamer geworden und gefüllt und stark. Ich nehme an Leib und Kraft zu; mit einem Wort: ich bin jetzt, Gott sei Dank, ganz gesund, kann besser gehen und Hände und Füße frei gebrauchen. Nur sind die betroffenen Teile noch nicht wieder ganz so stark wie sie waren. Alles dies aber habe ich näehst Gott der Milch zu danken, wovon ich jeden Morgen frisch und kuhwarm etwa einen halben Liter trinke; statt Mittagessen genieße ich ebensoviel Milch mit 
circiter haurio; loco prandii tantundem cum similia cocti comedo, et iturus cubitum //. bibo, nec ulla me lubido capit allis accumbentem, dulci valetudinis fructu contentum. Natura certe paucis contenta, quae ordinavit. Haec observatio ut et aliis prospere eveniat, votorum est alterum.
Weißbrot gekocht; danach trinke ich etwa dreiviertel Liter kalte Milch. Abends um acht Uhr esse ich wieder einen halben Liter Milch mit Weißbrot gekocht und trinke noch einen viertel Liter vor dem Schlafengehen. Das genügt mir; seit sechs Monaten esse und trinke ich nichts anderes und habe auch keine Lust auf andere Dinge. Denn ich befinde mich dabei sehr wohl und weiß den Wert der Gesundheit zu schätzen. Demnach begnügt sich die Natur also mit Wenigem; es ist mein großer Wunsch, da $\beta$ diese meine Beobachtung auch anderen Menschen zugut kommen möchte.

Brunners Verdienst, die Milch als Heilmittel in die Medizin und speziell in die Therapie der Stoffwechselerkrankungen eingeführt zu haben, ist sehr bemerkenswert, da ihm keinerlei Anhaltspunkte und Erklärungen, die der heutigen «Laborwissenschaft» geläufig sind, zu Beginn seines Heilverfahrens bekannt waren. Er gelangte rein epagogisch zu richtigen Ergebnissen. Karrell hat über ein Jahrhundert später die mit seinem Namen verbundene Milchkur in die Therapie der Herz- und Kreislauferkrankungen eingeführt, wahrscheinlich ohne zu wissen, daß Johann Conrad Brunner diese Kur lange vor ihm angewandt und beschrieben hat. «Pulsum quem olim habui celerem, nunc tardum, plenum et fortem percipio...» schreibt Brunner, und verdeutlicht damit klar und eindringlich die kreislaufentlastende Wirkung, die er mit seiner Kur erzielte. Bei Brunners Nierensteinen hat es sich offensichtlich um Uratsteine gehandelt, die mit Uratablagerungen an anderen Stellen seines Körpers einhergingen. Brunner bewirkte durch seine konsequent durchgeführte Schonkost eine Umstimmung des Harnes nach der alkalischen Seite, so daß die vorher ausfallenden Steine in Lösung blieben, und verhinderte außerdem durch die purinfreie Ernährung jede Absetzung von Harnsäurekristallen in den Gelenken. Damit kam die Gicht zum Stillstand. Da die Milch alle zum Aufbau des Körpers benötigten Bestandteile enthielt, konnte er die Kur lange Zeit fortsetzen, ohne irgendwelchen Mangelerscheinungen zu unterliegen. Wichtig ist auch der Hinweis auf die Steigerung der Anfälle nach Alkoholgenuß und deren Ausbleiben nach Alkoholentzug. Daß das Auftreten von Hämorrhoiden ebenfalls in Zusammenhang mit Alkohol und üppiger Lebensweise zu bringen ist, ist heute jedem Arzt bekannt. Auch hier gelangte Brunner empirisch zu Resultaten, die Physiologie und Chemie heute erklärend bestätigen. 
Die mäßige Lebensweise, die Brunner sich selbst auferlegte, verordnete er vielen seiner Patienten, die sich seinen Vorschriften widerspruchslos und gern unterwarfen. Die Großherzogin von Toscana hielt auf seine vernünftige, abwartende Behandlungsart so große Stücke, daß sie Brunner in einem Schreiben um Empfehlung eines Schweizer praktischen Arztes bat, «der nach seiner Art und nicht allzu chymisch die Patienten behandle...» ${ }^{25}$. Zweifellos kannte Brunner aber nicht nur den Wert von lactovegetabiler Kost, Trink- und Badekuren, sondern wußte um den tiefen Zusammenhang von Physis und Psyche und wandte dieses Wissen auch an, wie uns folgende Geschichte lehrt, die uns durch Aepli überliefert ist: «Der berühmte Herr Dr. Brunner wurde zu einem der größten Fürsten Deutschlands berufen, der an einem mit Mangel an Eßlust und Kraftlosigkeit verbundenen, und gegen alle Arzneyen widerspenstigen, schleichenden Fieber darnieder lag. Als Herr Brunner vernahm, daß der Kranke, dem von jeher die rohen Speisen seine Lieblingskost waren, einen großen Widerwillen gegen die zärtliche Kost, die ihm aufgezwungen wurde, bezeigte, so frug er ihn, ob er nicht Lust zu Sauerkraut hätte ? Bey dem Wort Sauerkraut lebte der Fürst auf. Seine Sehnsucht darnach war so groß, daß er die Zeit nicht erwarten konnte, bis es gekocht, und der Tisch gedeckt war. Zum Glück war ein solches für den Tisch der Stallknechte schon bereit. Herr Brunner ließ die hochaufgethürmte Schüssel ohne Ceremonien ins Fürstenzimmer bringen. Der Geruch labte ihn schon von weitem, und der Genuß desselben, und dergleichen Speisen stellten die Eßlust und die Kräfte dergestalt wieder her, daß nur wenige Arzneyen die gänzliche Genesung bewirken konnten. ${ }^{26}$

Dennoch hat Brunner nicht nur diätetische und balneologische Therapie angewandt. Er verließ sich solange auf sie, als er den spezifischen Erreger und das spezifische Heilmittel einer Krankheit nicht kannte. Brunner glaubte fest sowohl an das eine wie auch an das andere, und wir lesen heute mit Staunen, daß er dank der, allerdings damals noch sehr jungen und am Anfang ihrer großen Entwicklung stehenden, Erfindung des Mikroskopes, schon mit Sicherheit eine belebte Erregerwelt annahm. Über hundert Jahre vor Ehrenberg und über zweihundert Jahre vor der Entdeckung ScHauDinns und Hoffmanns schien ihm als Ursache der Lues ein «belebtes Wesen» oder «Würmchen» wahrscheinlich, das sich mit Quecksilber abtöten ließ, ebenso wie Läuse und Krätzemilben ${ }^{27}$. Auch für die Ruhr nahm

25 Aepli, S. 496.

26 Aepli, S. 576-7.

27 Nach Aepli. 
Magnifice Drg Retor

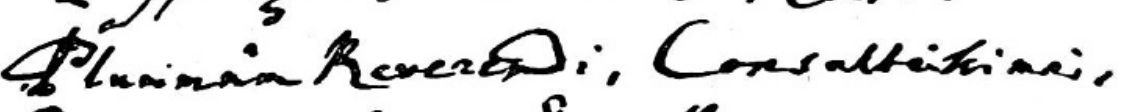

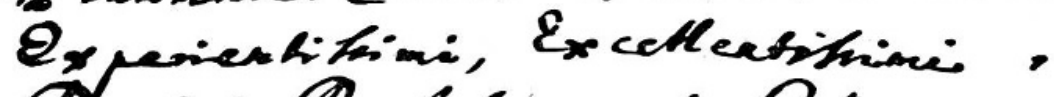

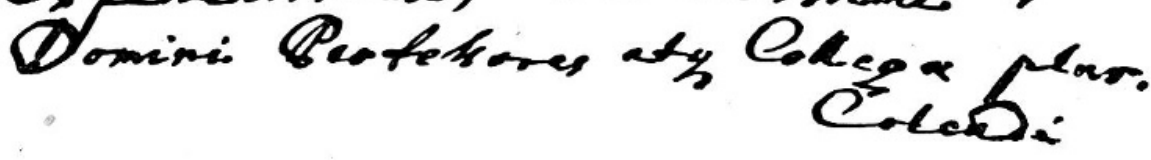

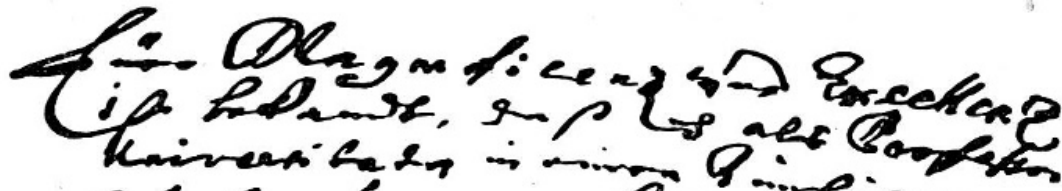

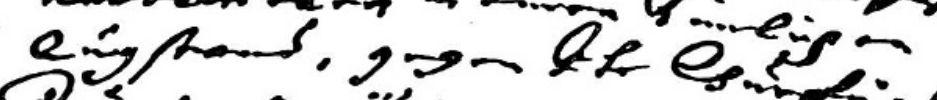

$$
\begin{aligned}
& Q^{2}=49 \text { s. }
\end{aligned}
$$

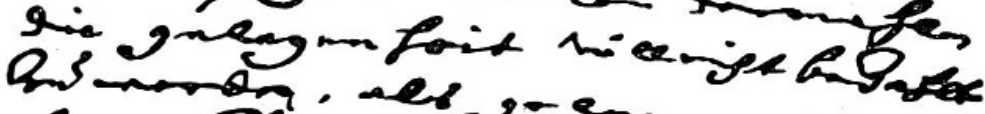

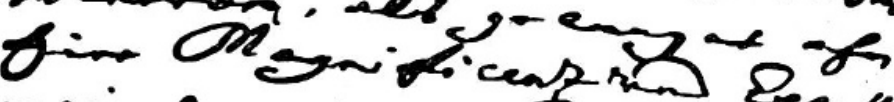

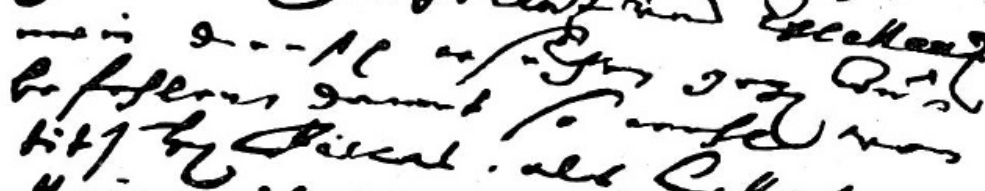

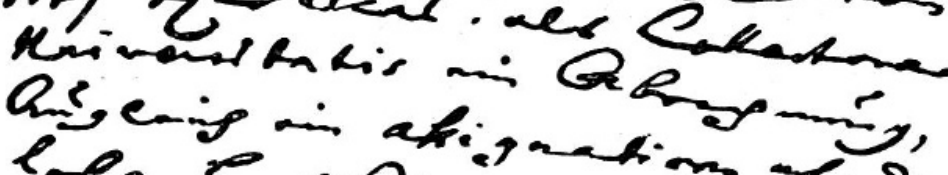

$$
\begin{aligned}
& \text { lic sen }
\end{aligned}
$$

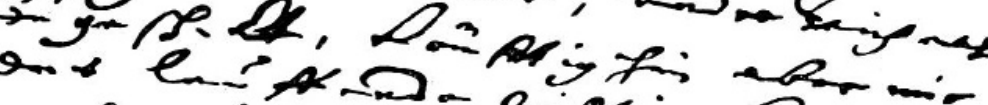

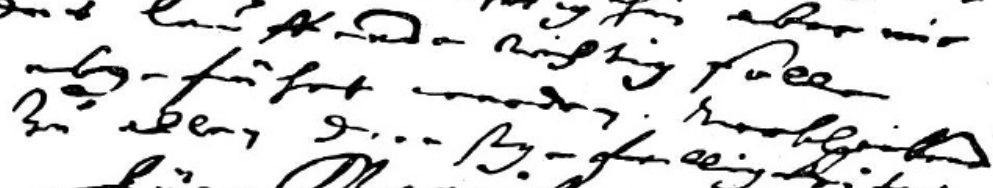

$$
\begin{aligned}
& \text { Cins Dingunen }
\end{aligned}
$$

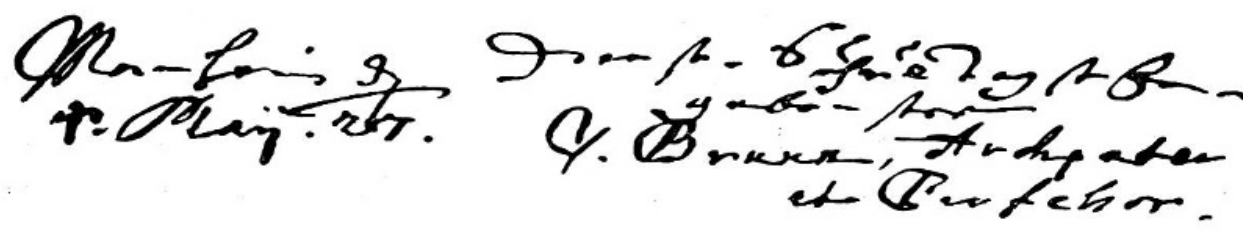

Abb. 4. Reklamationsschreiben über Gehaltsfragen an den Rektor der Universität Heidelberg, geschrieben in Mannheim 1727, ein halbes Jahr vor Brunners Tod 
er das Vorhandensein eines Contagiums an. «Contagium fuisse et $\mu i \alpha \sigma \mu \nu$ quod athmossphaeram, in qua degimus, perspiravit, et corpora humana pervasit, ex supra dictis patet; epidemice quippe grassabatur... ${ }^{28}$ Die klinischen Symptome der Ruhr hat Brunner kurz und treffend zusammengefaßt: «Excreta mucosa, cruore permixta, tenesmus ${ }^{29}$. Als Therapeutica gab er Ipecacuanha und Opiate.

Um bei einer ruhrerkrankten Wöchnerin die Übertragung der Krankheit von der Mutter auf das Kind durch Stillen zu verhindern, riet Brunner der Frau, das Kind zu entwöhnen. Die Milch ließ er durch junge Hunde aussaugen, die auch bald danach starben. Als die Wöchnerin nach einiger Zeit genesen war, ließ er das Kind ohne Bedenken wieder anlegen. - Eine andere ruhrerkrankte Wöchnerin weigerte sich, ihr Kind abzustillen. Die Krankheit übertrug sich schnell, und das Kind starb unter furchtbaren Krämpfen ${ }^{30}$. An spezifischen Mitteln verwandte Brunner in erster Linie die Chinarinde, die sich zunächst gegen viele Angriffe und Widerstände durchzusetzen hatte. Brunner erkannte früh die ausgezeichnete Wirkung, die diesem Mittel gegen alle entzündlichen und fieberhaften Erkrankungen innewohnte, und trug nicht wenig dazu bei, das Mißtrauen der Ärzteschaft zu verringern und dadurch der Chinarinde zu weiter Anerkennung und Verwendung zu verhelfen.

Bei Hochdruckerkrankungen, bei denen ein apoplektischer Anfall zu befürchten stand, entschloß sich Brunner gern zum Aderlaß, ungeachtet der Meinung seiner Kollegen, die sich dieser natürlichen Entlastungsmethode für den überbeanspruchten Kreislauf nicht mehr bedienten.

Es ist bezeichnend für Brunners vorsichtige, klug vorbeugende Behandlungsart, da $\beta$ er allen Neuerungen, die sich zur Aufgabe gemacht hatten, die Krankheits- und Ansteckungsgefahr einzuschränken, voll aufgeschlossen war. So erfahren wir aus einem Bericht, den Hugo aus Hannover an Brunner am 17. Juni 1724 schickte, daß er auf seinen Rat den Prinzen von Hannover der Pockenimpfung unterzogen habe, mit folgendem Verlauf: «Am elften Tage erfolgte der Ausbruch, dem zwey Tage lang ein erträgliches Fieber vorangieng. Im Gesichte befanden sich fünfzig Pusteln, und in allem am ganzen Leibe kaum fünfhundert, alle sehr vollkommen, und hinterließen keine Narben. Die geringen Einschnitte an beyden Armen, wobey nur das Oberhäutchen getrennt wurde, hatten bald nach beygebrachtem

${ }^{28}$ Experimentum dysenteriae, Obs. 206, Eph. Decur. II, Ann. VI, S. 387.

${ }^{29}$ Ibidem.

${ }^{30}$ Nach Aepli, S. 538. 
Pockengift zu eitern angefangen, und flossen während der ganzen Krankheit mehr oder weniger. Der Prinz befinde sich herrlich wohl.» ${ }^{31}$

Offensichtlich war Brunner die in England und besonders von TномаS Sydenham seit kurzem geübte Methode der Pockenimpfung - nicht mit Vaccine sondern mit Pustelinhalt - bekannt, wenn wir auch leider nicht erfahren können, ob und wo er sie sonst angewandt hat.

So abwartend internistisch Brunner im allgemeinen therapeutisch vorzugehen pflegte, so rasch griff er chirurgisch ein, wenn er es für unumgänglich notwendig hielt. Dies beweist uns seine Behandlung bei Ascites. Bei einer 49 jährigen Frau, die er zunächst - erfolglos - mit verschiedenen Hausmitteln behandelt hatte, nahm der Ascites in solchem $\mathrm{Maß}$ zu, daß Brunner sich entschloß, unter den obwaltenden Umständen eine Parazentese vorzunehmen. Er machte drei Finger unterhalb des Nabels seitlich einen kleinen Einschnitt mit der Lanzette, hielt die Wunde über drei Wochen offen, und ließ während dieser Zeit dreizehn Liter Wasser ab. Nach anfänglichem Fieber erholte sich die Patientin bald, so daß er die Nachbehandlung einem Chirurgen überlassen konnte.

Es wäre müßig, alle erfolgreichen Kuren, die Brunner im Laufe seines Lebens begonnen und durchgeführt hat, aufzuzählen, ebenso müßig, wie die Unzahl von Dekokten seiner Apotheke nennen zu wollen. Sie sind für die heutige Medizin von geringer Bedeutung, gemessen an den viel wesentlicheren Hinweisen, die uns seine Diätetik gegeben hat.

Brunner hat seiner Arbeit über das Pankreas den Satz Senecas : «Multum egerunt, qui ante nos fuerunt», vorangestellt. Der Satz ist unwandelbar. Nur die Persönlichkeit, der die ehrfürchtige Verneigung gilt, wechselt mit dem Betrachter. «Multum egerunt, qui ante nos fuerunt»; Johann Conrad Brunner hat unendlich viel dazu getan, das Haus der Medizin bauen und vollenden zu helfen.

\section{Literatur}

J.M. AEPLI, «Dr. Johann Conrad Brunner», in Archiv gemeinnütz. phys. und med. Kenntnis. I, 2, Zürich 1787 (zit. Aepli).

C. Brunner, «Dr. Johannes Conrad Brunner», in Virchow und von HoltzendorfF, Gemeinverständliche Vorträge, N. F., Ser. 3, 62, Hamburg 1888.

C. Brunner und W. von Muralt, Aus den Briefen hervorragender Schweizer Ärzte des 17. Jahrhunderts, Schwabe, Basel 1919 (zit. Brunner und von Muralt).

F. HoFF, Behandlung innerer Krankheiten, 3. Auflage, Leipzig 1948.

${ }^{31}$ Aepli, S. 517. 
P. KüBler, Geschichte der Pocken und der Impfung, Berlin 1901.

D. W. Nebel, «Oratio de vita et meritis Professorum Medicinae...», in Acta Sacrorum Secularium, Heidelberg 1787, p. 253.

J.J. Scheuchzer, Memoria Brunneriana. Eph. nat. cur., Appendix, Band IV, 1737 (zit. Scheuchzer).

Јон. Sсншав, Quatuor seculorum syllabus Rectorum, Heidelberg 1786 (zit. Schwab).

E. Stüвler, Geschichte der medizinischen Fakultät der Universität Heidelberg, Heidelberg 1926.

E. Winkelmann, Urkundenbuch der Universität Heidelberg, Heidelberg 1886 (zit. Winkelmann).

G. Wolf-HeIdegger, «Johann Conrad Brunner», in Große Schweizer Forscher, AtlantisVerlag, Zürich 1939.

O. Chr. Zimmermann, «Die erste Beschreibung von Symptomen des experimentellen Pankreas-Diabetes durch den Schweizer Joh. Conr. Brunner (1653-1727)», in Gesnerus 2 (1945) Heft 3. 